\title{
Power Minimization of UAV-Assisted Full-Duplex Mobile Relaying via Hybrid Trajectory Design
}

Xiaodong Ji ( $\sigma_{\text {jxd@ntu.edu.cn ) }}$

Nantong University Wenfeng Campus https://orcid.org/0000-0002-1324-8051

Tao Wang

Nantong University

Chong Qian

China Telecom

Chenhao Huang

China Telecom

\section{Research}

Keywords: UAV communication, trajectory design, power minimization

Posted Date: September 29th, 2021

DOI: https://doi.org/10.21203/rs.3.rs-915825/v1

License: (9) This work is licensed under a Creative Commons Attribution 4.0 International License.

Read Full License 


\title{
Power Minimization of UAV-Assisted Full-Duplex Mobile Relaying via Hybrid Trajectory Design
}

\author{
Xiaodong Ji ${ }^{*}{ }^{1}$, Tao Wang ${ }^{1}$, Chong Qian ${ }^{2}$, Chenhao Huang ${ }^{3}$ \\ 1. School of Information Science and Technology, Nantong University, Nantong 226019, China \\ 2. Wujiang Branch of China Telecom Group Co., Suzhou 215200, China \\ 3. Gaoyou Branch of China Telecom Group Co., Yangzhou 225600, China \\ * Corresponding author: Xiaodong Ji, E-mail: jxd@ntu.edu.cn
}

Tao Wang, E-mail: 1290549851@qq.com

Chong Qian, E-mail: 865506980@qq.com

Chenhao Huang, E-mail: 121900180@qq.com

\begin{abstract}
This paper solved a propulsion power minimization problem subject to a total data-bits constraint of an unmanned aerial vehicle (UAV) enabled full-duplex mobile relaying system, where a full-duplex UAV relay is dispatched as a mobile relay to assist data transfer from a source to a destination by using three trajectory flying modes, namely, the UAV first flies in a circle above the source, next flies to the destination in a straight line, and finally flies in a circle above the destination until all the data bits has been transferred. Since the propulsion power minimization problem is a non-convex mixed integer programming problem and its closed-form solution is hard to obtain, it is transformed to three sub-problems so as to simplify its solution. After solving the three sub-problems, an iterative algorithm is proposed to achieve a sub-optimal solution to the propulsion power minimization problem, leading to a new hybrid circular/straight trajectory (HCST) design. Computer simulations are conducted and the results validated the proposed HCST design. It is shown that compared to the straight or circular flight trajectory design, the HCST performs well in terms of energy saving for the long distance and big data communication cases.
\end{abstract}

Index terms - UAV communication, trajectory design, power minimization

\section{Introduction}

The use of unmanned aerial vehicles (UAVs) for assisting wireless communications has attracted increasing interest recently [1]. Compared to the traditional terrestrial communications, UAV-assisted communications are more cost-effective by enabling on-demand operations, more flexible and swift for deployment due to the high mobility of UAVs. In addition, UAV-ground communication links are more likely to have line-of-sight (LoS) channels which offers higher capacity than that provided by terrestrial links. Thus, UAVs shall play an extremely important role in future wireless communications with applications such as temporary traffic offloading for cellular base stations [2]-[4], cooperative communications for mobile relaying [5]-[8], information dissemination 
and data collection for Internet of Things [9], etc.

In this paper, we considered a full-duplex mobile relaying system consisting of a fixed-wing UAV, a source and a destination. The source would like to transfer data to the destination assisted by the UAV which acts as a decode-and-forward (DF) relay. The UAV relay has three trajectory flying modes, namely, the UAV first flies in a circle above the source, next flies to the destination in a straight line, and finally flies in a circle above the destination until all the data bits has been transferred. It is noted in [10] that an UAV's communication-related power is usually much smaller than its propulsion power. Therefore, the main goal of this paper is to minimize the total propulsion power of the UAV. We formulate the trajectory design as a non-convex mixed integer programming problem. To solve the problem, it is dived into a few sub-problems, by solving which a sub-optimal solution is acquired. Then, an iterative algorithm is proposed to obtain a hybrid circular/straight trajectory (HCST) design which can approach to the optimal trajectory minimizing the total propulsion power.

The rest of this paper is organized as follows: Section 2 describes the related works in literature. Section 3 introduces the system model under investigation and formulates a general optimization problem corresponding to the propulsion power minimization. By dividing the optimization problem into three sub-problems, the power minimization problem is solved in Section 4, leading to a new HCST design. Computer simulations of the proposed HCST design with performance comparisons are presented in Section 5. Section 6 concludes the paper.

\section{Related works}

Currently, there are many works in literature that concern the problem of maximizing information throughput or spectrum efficiency of UAV-assisted mobile relaying systems [11]-[16]. It is known that UAVs have limited on-board energy, and thus energy saving has been recognized as a more important factor for UAV communications. The authors of [17] investigated an energy-efficiency problem by optimizing the UAV's speed and load factors in circular trajectory. In [18], an energy-efficiency maximization problem was solved by jointly studying beamforming, power allocation, circular radius and flight speed of an UAV-assisted half-duplex mobile relaying system. In [19], a power consumption trade-off between ground terminal and UAVs was derived for circular and straight trajectories, respectively. The authors of [10] developed a load-carry-and-delivery scheme for a full-duplex UAV relaying network with the aim of enhancing the energy efficiency of the network. In [20], a rotary-wing UAV was used to communicate with multiple ground nodes, and an efficient algorithm was proposed to optimize the hovering locations and duration, as well as the flying trajectory so as to minimize the system energy consumption. The authors of [21] investigated an energy-efficiency problem with the aim of maximizing the data bits transferred per the UAV's propulsion energy via the trajectory design. It should be noted that most 
of the existing works considered the circular or straight trajectory design separately. It is noted in [21] that there exists an upper bound of information throughput if a straight trajectory is adopted. It means that straight trajectory is not suitable for the case where a huge amount of data need to be transferred. In case that a circular trajectory is employed, the system information throughput will be poor if the source and destination nodes are located far away [17]. In view of the shortcomings of circular and straight trajectories, this paper investigates a HCST design for power minimization.

\section{Method}

Fig. 1 gives the system model, where an UAV denoted by $\mathrm{R}$ is dispatched as a mobile relay to assist data transfer from the source S to the destination D. Suppose that S and D are located far away, and thus no direct link exists between S and D due to high path loss caused by long distance and/or obstacles. Moreover, it is assumed that $\mathrm{S}$ would like to transfer a huge amount of data to $\mathrm{D}$.

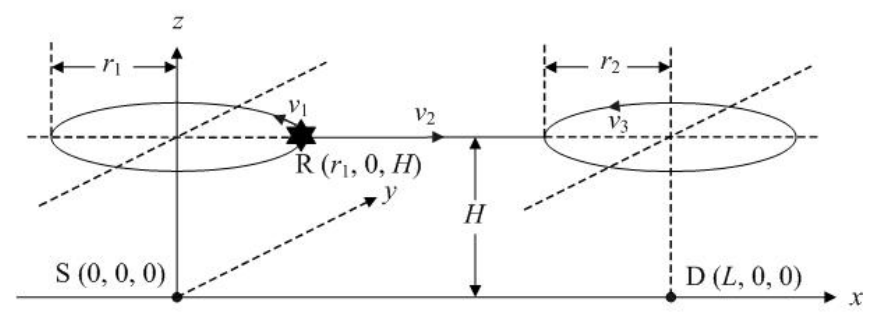

Fig. 1 UAV-enabled mobile relaying system with hybrid circular/straight trajectory

Without loss of generality, assume that the horizontal coordinates of $\mathrm{S}$ and $\mathrm{D}$ are $\mathbf{q}_{\mathrm{S}}=[0,0]^{\mathrm{T}}$ and $\mathbf{q}_{\mathrm{D}}=[L, 0]^{\mathrm{T}}$, respectively, and the UAV flies at a fixed altitude $H$ which corresponds to the minimum altitude required for terrain or building avoidance without frequent aircraft ascending or descending [10]. Denote by $T$ the mission completion time. Throughout the whole mission time, the UAV needs to collect $Q$ bits data from $\mathrm{S}$ and then forwards them to D. There exists three trajectory modes described as the following.

Mode 1: The UAV flies in a circle with center at $(0,0, H)$ and radius $r_{1}$. Denote by $v_{1}$ and $T_{1}$ the UAV's speed and duration of mode 1 , respectively.

Mode 2: The UAV flies from $\left(r_{1}, 0, H\right)$ to $\left(L-r_{2}, 0, H\right)$ in a straight line. Denote by $v_{2}$ and $T_{2}$ the UAV's speed and duration of mode 2, respectively.

Mode 3: The UAV flies in a circle with center at $(L, 0, H)$ and radius $r_{2}$. Denote by $v_{3}$ and $T_{3}$ the UAV's speed and duration of mode 3 , respectively.

Suppose that the initial location of the UAV is $\left(r_{1}, 0, H\right)$. First, the UAV flies $n$ laps according to mode 1 . Then, the UAV flies along mode 2. Finally, the UAV flies according to mode 3 until $Q$ bits data are completely transferred from $\mathrm{S}$ to D. Here, the duration of each mode must satisfy

$$
T_{1}=\frac{2 \pi r_{1}}{v_{1}} n,(n=1,2, \cdots), T_{2}=\frac{L-r_{1}-r_{2}}{v_{2}}, T_{3} \geq 0 .
$$

During the whole mission time, a full-duplex relaying fashion is employed, namely, the UAV collects data from $\mathrm{S}$ and forwards them to D simultaneously. Note that for simplicity, the UAV's take-off and landing phases are 
ignored. It is worth-mentioning that the UAV's trajectory should change between different modes smoothly, and the UAV's speed cannot be switched instantly between $v_{1}, v_{2}$ and $v_{3}$. However, since the total power consumption of an UAV is very large, the change of the UAV's kinetic energy between speeds $v_{1}, v_{2}$ and $v_{3}$ and the difference between the practical trajectory and the trajectory in Fig. 1 can be omitted. Denote by $\mathbf{q}(t) \in \mathbf{R}^{2 \times 1}$ the UAV's trajectory on the horizontal coordinate, where $0 \leq t \leq T$. Then, the time-varying distance between $\mathrm{S}$ and the UAV $\mathrm{R}$, and that between the UAV R and D can be, respectively, expressed as

$$
\begin{gathered}
d_{\mathrm{SR}}(t)=\sqrt{H^{2}+\|\mathbf{q}(t)\|^{2}}, \\
d_{\mathrm{RD}}(t)=\sqrt{H^{2}+\left\|(L, 0)^{T}-\mathbf{q}(t)\right\|^{2}} .
\end{gathered}
$$

Here, it is assumed that the UAV $\mathrm{R}$ is equipped with a data-buffer of sufficiently large size and the communication links from $\mathrm{R}$ to $\mathrm{S}$ and $\mathrm{D}$ are dominated by the Line of Sight channels [11]. Moreover, the Doppler effect due to the UAV mobility is assumed to be perfectly compensated [11]. Then, the time-varying channel between $\mathrm{S}$ and $\mathrm{R}$ follows the free-space path loss model and can be written as

$$
h_{\mathrm{SR}}(t)=\beta_{0} d_{\mathrm{SR}}^{-2}(t)=\frac{\beta_{0}}{H^{2}+\|\mathbf{q}(t)\|^{2}} .
$$

Likewise, the time-varying channel between $\mathrm{R}$ and $\mathrm{D}$ can be expressed as

$$
h_{\mathrm{RD}}(t)=\beta_{0} d_{\mathrm{RD}}^{-2}(t)=\frac{\beta_{0}}{H^{2}+\left\|(L, 0)^{T}-\mathbf{q}(t)\right\|^{2}} .
$$

In (4) and (5), $\beta_{0}$ denotes the channel power at the reference distance $d_{0}=1 \mathrm{~m}$. Assume that $\mathrm{R}$ collects and forwards data using different frequency bands, and thus there is no loop-interference at the UAV[11]. Then, the corresponding signals received by $\mathrm{R}$ and $\mathrm{D}$ can be, respectively, written as

$$
\begin{aligned}
& y_{\mathrm{R}}(t)=\sqrt{P_{\mathrm{S}} h_{\mathrm{SR}}(t)} x_{\mathrm{S}}(t)+n_{\mathrm{R}}(t), \\
& y_{\mathrm{D}}(t)=\sqrt{P_{\mathrm{R}} h_{\mathrm{RD}}(t)} x_{\mathrm{R}}(t)+n_{\mathrm{D}}(t) .
\end{aligned}
$$

In (6) and (7), $x_{\mathrm{S}}(t)$ and $x_{\mathrm{R}}(t)$ are the signals sent by $\mathrm{S}$ and $\mathrm{R} ; P_{\mathrm{S}}$ and $P_{\mathrm{R}}$ are the transmit power from $\mathrm{S}$ and $\mathrm{R} ; n_{\mathrm{R}}(t)$ and $n_{\mathrm{D}}(t)$ are the additive noises received by $\mathrm{R}$ and $\mathrm{D}$, respectively.

The data of size $Q_{\mathrm{R}}(t)$ and $Q_{\mathrm{D}}(t)$ received by the UAV R and D at time $t$ in bits/Hz can be, respectively, expressed as [10]

$$
\begin{gathered}
Q_{\mathrm{R}}(t)=\int_{0}^{t} \log _{2}\left(1+\frac{P_{\mathrm{S}} h_{\mathrm{SR}}(\tau)}{\sigma^{2}}\right) d \tau=\int_{0}^{t} \log _{2}\left(1+\frac{P_{\mathrm{S}} \gamma_{0}}{H^{2}+\|\mathbf{q}(\tau)\|^{2}}\right) d \tau, \\
Q_{\mathrm{D}}(t)=\int_{0}^{t} \log _{2}\left(1+\frac{P_{\mathrm{R}} h_{\mathrm{RD}}(\tau)}{\sigma^{2}}\right) d \tau=\int_{0}^{t} \log _{2}\left(1+\frac{P_{\mathrm{R}} \gamma_{0}}{H^{2}+\left\|(L, 0)^{\mathrm{T}}-\mathbf{q}(\tau)\right\|^{2}}\right) d \tau,
\end{gathered}
$$

where $\sigma^{2}$ denotes the noise power at the UAV R and D, and $\gamma_{0} \stackrel{\Delta}{=} \beta_{0} / \sigma^{2}$. It is worth-mentioning that in the paper, data bits are normalized by bandwidth, and thus without specific instructions, bit means bit/Hz. In order to ensure $Q$ bits data transfer during mission completion time $T,(10)$ must be satisfied. 


$$
Q_{\mathrm{R}}(T) \geq Q, Q_{\mathrm{D}}(T) \geq Q
$$

Moreover, the UAV can only forward data that has already been collected from S, and thus the following information-causality constraint must be satisfied [20]

$$
Q_{\mathrm{D}}(t) \leq Q_{\mathrm{R}}(t), 0 \leq t \leq T .
$$

It is well known that the total power consumption of an UAV consists of two parts, i.e., communication-related and propulsion power consumption. Here, only the UAV's propulsion power consumption is considered as the communication-related power consumption is usually much smaller than the propulsion power consumption [10]. According to [19], propulsion power consumption of a fixed-wing UAV with circular and straight trajectories can be modeled as $\left[c_{1}+c_{2} /\left(\mathrm{g}^{2} r^{2}\right)\right] v^{3}+c_{2} / v$ and $c_{1} v^{3}+c_{2} / v$, respectively. Here, $c_{1}$ and $c_{2}$ are the parameters depending on the aircraft's weight, wing area, air density, etc., and $g, r$ and $v$ denote the acceleration of gravity, the radius of the circular and the velocity of the UAV, respectively [19]. Then, the optimization problem corresponding to the propulsion power minimization can be formulated as

$$
\begin{array}{rl}
\left(r_{1}^{*}, r_{2}^{*}, v_{1}^{*}, v_{2}^{*}, v_{3}^{*}, T_{1}^{*}, T_{2}^{*}, T_{3}^{*}, n^{*}\right)=\arg \min _{r_{1}, r_{2}, v_{1}, v_{2}, v_{3}, T_{1}, T_{2}, T_{3}, n} & T\left(\left[c_{1}+\frac{c_{2}}{g^{2} r_{1}^{2}}\right) v_{1}^{3}+\frac{c_{2}}{v_{1}}\right]+T_{2}\left(c_{1} v_{2}^{3}+\frac{c_{2}}{v_{2}}\right) \\
& +T_{3}\left[\left(c_{1}+\frac{c_{2}}{g^{2} r_{2}^{2}}\right) v_{3}^{3}+\frac{c_{2}}{v_{3}}\right] \\
\text { s.t. } \quad & r_{1}+r_{2} \leq L, r_{1}>0, r_{2}>0 \\
& V_{\text {min }} \leq v_{1}, v_{2}, v_{3} \leq V_{\max } \\
& T_{1}=\frac{2 \pi r_{1}}{v_{1}} n,(n=1,2, \cdots), T_{2}=\frac{L-r_{1}-r_{2}}{v_{2}}, T_{3} \geq 0 \\
& Q_{\mathrm{R}}(T) \geq Q, Q_{\mathrm{D}}(T) \geq Q \\
& Q_{\mathrm{D}}(t) \leq Q_{\mathrm{R}}(t), 0 \leq t \leq T \\
& T_{1}+T_{2}+T_{3}=T
\end{array}
$$

where $V_{\min }$ and $V_{\max }$ are the UAV's the minimum and maximum flight velocity constraints, and (12b) and (12c) are the practical physical constraints.

\section{Solution of Power Minimization Problem}

In this section, the power minimization problem (12) is solved, leading to a HCST design. As a first step, the following lemma is presented so as to simplify the optimization problem.

Lemma 1: For problem (12), the constraints (12e) and (12f) can be rewritten as

$$
Q_{\mathrm{R}}(T) \geq Q_{\mathrm{D}}(T)=Q
$$

Proof: It is noticed that $Q_{\mathrm{R}}(t)$ and $Q_{\mathrm{D}}(t)$ are both monotonically increasing functions for $t \in[0,+\infty)$. Moreover, when $\mathrm{S}$ and $\mathrm{D}$ are located far away, $Q_{\mathrm{R}}(t)$ can be seen as a convex function about $t$, while $Q_{\mathrm{D}}(t)$ is a concave function. Since $Q_{\mathrm{R}}(0)=Q_{\mathrm{D}}(0)=0,(12 \mathrm{f})$ can be satisfied if $Q_{\mathrm{R}}(T) \geq Q_{\mathrm{D}}(T)$ holds. Assume that $Q_{\mathrm{D}}\left(T^{*}\right)>Q$ holds, where $T^{*}=T_{1}^{*}+T_{2}^{*}+T_{3}^{*}$ and $T_{1}^{*}, T_{2}^{*}$ and $T_{3}^{*}$ are the solutions of (12). Then, there must 
exist a $\Delta>0$ such that $T_{1}^{*}, T_{2}^{*}$ and $T_{3}^{*}-\Delta$ can satisfy the constraints, and further reduces the power consumption, which contradicts the optimality of $T_{1}^{*}, T_{2}^{*}$ and $T_{3}^{*}$. Therefore, the optimal solution of (12) must satisfy $Q_{\mathrm{D}}\left(T^{*}\right)=Q$, and (12e) and (12f) can be rewritten as $Q_{\mathrm{R}}(T) \geq Q_{\mathrm{D}}(T)=Q$.

Denote by $Q_{\mathrm{R}}^{\text {cirl }}, Q_{\mathrm{R}}^{\mathrm{str}}$ and $Q_{\mathrm{R}}^{\text {cir2 }}$ the sizes of data bits received by the UAV in the duration of modes 1,2 and 3, respectively. Denote by $Q_{\mathrm{D}}^{\text {cir1 }}, Q_{\mathrm{D}}^{\text {str }}$ and $Q_{\mathrm{D}}^{\text {cir2 }}$ the sizes of data bits received by D in the duration of modes 1,2 and 3, respectively. Then, (13) can be rewritten as

$$
Q_{\mathrm{R}}^{\text {cirl }}+Q_{\mathrm{R}}^{\mathrm{str}}+Q_{\mathrm{R}}^{\text {cir2 }} \geq Q_{\mathrm{D}}^{\text {cir1 }}+Q_{\mathrm{D}}^{\mathrm{str}}+Q_{\mathrm{D}}^{\text {cir2 }}=Q,
$$

and $Q_{\mathrm{R}}^{\text {cirl }}$ can be expressed as

$$
Q_{\mathrm{R}}^{\text {cirl }}=\frac{2 n \pi r_{1}}{v_{1}} \log _{2}\left(1+\frac{P_{\mathrm{S}} \gamma_{0}}{H^{2}+r_{1}^{2}}\right)
$$

According to [19], $Q_{R}^{\text {str }}$ can be given by

$$
Q_{\mathrm{R}}^{\mathrm{str}}=\frac{F_{1}\left(L-r_{2}\right)-F_{1}\left(r_{1}\right)}{v_{2} \ln 2},
$$

where $F_{1}(x)=x \ln \left(1+\frac{P_{\mathrm{S}} \gamma_{0}}{H^{2}+x^{2}}\right)-2 H \tan ^{-1}\left(\frac{x}{H}\right)+2 \sqrt{H^{2}+P_{\mathrm{S}} \gamma_{0}} \tan ^{-1}\left(\frac{x}{\sqrt{H^{2}+P_{\mathrm{S}} \gamma_{0}}}\right)$.

Since $Q_{\mathrm{R}}^{\mathrm{cir} 2}$ is mathematically intractable, a corresponding lower bound is considered. It should be noted that a lower bound of $Q_{\mathrm{R}}^{\text {cir2 }}$ satisfying (14) can enable (12e) and (12f) to be hold. Using inequality (52) in [10], $Q_{\mathrm{R}}^{\text {cir2 }}$ can be lower-bounded as

$$
Q_{\mathrm{R}}^{\mathrm{cir} 2}=\int_{-\pi}^{\frac{v_{3}}{r_{2}} T_{3}-\pi} \frac{r_{2}}{v_{3}} \log _{2}\left(1+\frac{P_{\mathrm{S}} \gamma_{0}}{H^{2}+L^{2}+2 L r_{2} \cos x+r_{2}^{2}}\right) d x \geq T_{3} B_{1}\left(r_{2}\right)+B_{2}\left(r_{2}, v_{3}\right),
$$

where functions $B_{1}(x)$ and $B_{2}(x, y)$ are given as the following.

$$
\begin{gathered}
B_{1}(x)=\log _{2}\left(1+\frac{P_{\mathrm{S}} \gamma_{0}}{H^{2}+L^{2}+x^{2}}\right), \\
B_{2}(x, y)=-\frac{2\left(\log _{2} e\right) P_{\mathrm{s}} \gamma_{0} L x^{2}}{y\left(H^{2}+L^{2}+x^{2}\right)\left(H^{2}+L^{2}+x^{2}+P_{\mathrm{S}} \gamma_{0}\right)} .
\end{gathered}
$$

Using the same approach, it is readily to obtain $Q_{\mathrm{D}}^{\text {cirl }} \geq T_{1} B_{3}\left(r_{1}\right)+B_{4}\left(r_{1}, v_{1}\right), \quad Q_{\mathrm{D}}^{\mathrm{str}}=\frac{F_{2}\left(L-r_{1}\right)-F_{2}\left(r_{2}\right)}{v_{2} \ln 2}$ and $Q_{\mathrm{D}}^{\text {cir2 }}=T_{3} \log _{2}\left(1+\frac{P_{\mathrm{R}} \gamma_{0}}{H^{2}+r_{2}^{2}}\right)$, where functions $B_{3}(x), B_{4}(x, y)$ and $F_{2}(x)$ are defined as the following.

$$
\begin{gathered}
B_{3}(x)=\log _{2}\left(1+\frac{P_{\mathrm{R}} \gamma_{0}}{H^{2}+L^{2}+x^{2}}\right), \\
B_{4}(x, y)=-\frac{2\left(\log _{2} e\right) P_{\mathrm{R}} \gamma_{0} L x^{2}}{y\left(H^{2}+L^{2}+x^{2}\right)\left(H^{2}+L^{2}+x^{2}+P_{\mathrm{R}} \gamma_{0}\right)} \\
F_{2}(x)=x \ln \left(1+\frac{P_{\mathrm{R}} \gamma_{0}}{H^{2}+x^{2}}\right)-2 H \tan ^{-1}\left(\frac{x}{H}\right)+2 \sqrt{H^{2}+P_{\mathrm{R}} \gamma_{0}} \tan ^{-1}\left(\frac{x}{\sqrt{H^{2}+P_{\mathrm{R}} \gamma_{0}}}\right) .
\end{gathered}
$$


Since problem (12) is a non-convex mixed integer programming problem, it cannot be directly solved with standard convex optimization techniques. In the following, problem (12) is divided into three sub-problems by solving which a sub-optimal solution to the power minimization problem is acquired.

Recall that S and D are located far away and the UAV relay assists the data transfer between $\mathrm{S}$ and D by adopting three trajectory modes. In mode 1 , the UAV is much closer to $\mathrm{S}$ than to $\mathrm{D}$, and thus it has dominant traffic volume in collecting data from the source S. On the contrary, in mode 3 the traffic of the link from the UAV to D is dominant. Therefore, in order to simplify (12), two sub-problems corresponding to the power minimization in modes 1 and 3 are addressed first, where only the link having the dominant traffic volume is considered. Then, the two sub-problems can be formulated as (18) and (19).

$$
\begin{aligned}
\left(\tilde{r}_{1}, \tilde{v}_{1}\right) & =\arg \min _{r_{1}, v_{1}} 2 \pi n\left[\left(c_{1} r_{1}+\frac{c_{2}}{g^{2} r_{1}}\right) v_{1}^{2}+\frac{c_{2} r_{1}}{v_{1}^{2}}\right] \\
\text { s.t. } \quad Q_{\mathrm{R}}^{\text {cir1 }} & =\frac{2 n \pi r_{1} \log _{2}\left(1+\frac{P_{\mathrm{S}} \gamma_{0}}{H^{2}+r_{1}^{2}}\right)}{v_{1}} \\
v_{\min } & \leq v_{1} \leq v_{\max }, \quad 0<r_{1}<L \\
\left(\tilde{r}_{2}, \tilde{v}_{3}\right) & =\arg \min _{r_{2}, v_{3}} T_{3}\left[\left(c_{1}+\frac{c_{2}}{g^{2} r_{2}^{2}}\right) v_{3}^{3}+\frac{c_{2}}{v_{3}}\right] \\
\text { s.t. } \quad Q_{\mathrm{D}}^{\text {cir2 }} & =T_{3} \log _{2}\left(1+\frac{P_{\mathrm{R}} \gamma_{0}}{H^{2}+r_{2}^{2}}\right) \\
v_{\min } & \leq v_{3} \leq v_{\max }, 0<r_{2}<L, T_{3} \geq 0
\end{aligned}
$$

Of note is that in (18) and (19), parameters $n, Q_{\mathrm{R}}^{\text {cir1 }}$ and $Q_{\mathrm{D}}^{\text {cir2 }}$ are assumed to be fixed.

For problem (18), the optimal radius $\tilde{r}_{1}$ and velocity $\tilde{v}_{1}$ can be obtained via one-dimensional search by substituting (18b) into (18a). For problem (19), substituting (19b) into (19a) leads to

$$
\begin{aligned}
& \left(\tilde{r}_{2}, \tilde{v}_{3}\right)=\arg \min _{r_{2}, v_{3}} \frac{\left(c_{1}+\frac{c_{2}}{g^{2} r_{2}^{2}}\right) v_{3}^{3}+\frac{c_{2}}{v_{3}}}{\log _{2}\left(1+\frac{P_{\mathrm{R}} \gamma_{0}}{H^{2}+r_{2}^{2}}\right)} \\
& \text { s.t. } \quad v_{\min } \leq v_{3} \leq v_{\max }, 0<r_{2}<L
\end{aligned}
$$

It can be easily found that $\left[c_{1}+c_{2} /\left(g^{2} r_{2}^{2}\right)\right] v_{3}^{3}+c_{2} / v_{3}$ is a concave function for $v_{3} \in(0,+\infty)$. It's extreme point is $v_{3}=\left\{c_{2} /\left[3 c_{1}+3 c_{2} /\left(g^{2} r_{2}^{2}\right)\right]\right\}^{\frac{1}{4}}$. So, the minimum power on the numerator of (20a) can be achieved when (21) holds. 


$$
\tilde{v}_{3}=\left\{\begin{array}{l}
v_{\text {min }} \text {, if } v_{\text {min }}>\left(\frac{c_{2} g^{2} r_{2}^{2}}{3 c_{1} g^{2} r_{2}^{2}+3 c_{2}}\right)^{\frac{1}{4}} \\
\left(\frac{c_{2} g^{2} r_{2}^{2}}{3 c_{1} g^{2} r_{2}^{2}+3 c_{2}}\right)^{\frac{1}{4}} \text {,if } v_{\min } \leq\left(\frac{c_{2} g^{2} r_{2}^{2}}{3 c_{1} g^{2} r_{2}^{2}+3 c_{2}}\right)^{\frac{1}{4}} \leq v_{\max } . \\
v_{\text {max }}, \text { if } v_{\max }<\left(\frac{c_{2} g^{2} r_{2}^{2}}{3 c_{1} g^{2} r_{2}^{2}+3 c_{2}}\right)^{\frac{1}{4}}
\end{array}\right.
$$

Substituting (21) into (20) and then the optimal solution of (20) can be obtained by using one-dimensional search over $0<r_{2}<L$.

Base on the above discussions, (12) can be transformed into

$$
\begin{array}{ll}
\left(\tilde{v}_{2}, \tilde{T}_{1}, \tilde{T}_{3}, \tilde{n}\right)=\arg \min _{v_{2}, T_{1}, T_{3}, n} T_{1}\left[\left(c_{1}+\frac{c_{2}}{g^{2} \tilde{r}_{1}^{2}}\right) \tilde{v}_{1}^{3}+\frac{c_{2}}{\tilde{v}_{1}}\right]+T_{2}\left(c_{1} v_{2}^{3}+\frac{c_{2}}{v_{2}}\right)+T_{3}\left[\left(c_{1}+\frac{c_{2}}{g^{2} \tilde{r}_{2}^{2}}\right) \tilde{v}_{3}^{2}+\frac{c_{2}}{\tilde{v}_{3}^{2}}\right] \\
\text { s.t. } \quad V_{\min } \leq v_{2} \leq V_{\max } \\
T_{1}=\frac{2 \pi \tilde{r}_{1}}{\tilde{v}_{1}} n,(n=1,2, \cdots), T_{2}=\frac{L-\tilde{r}_{1}-\tilde{r}_{2}}{v_{2}}, T_{3} \geq 0 \\
Q_{\mathrm{R}}^{\text {cirl }}+Q_{\mathrm{R}}^{\mathrm{str}}+B_{1}\left(\tilde{r}_{2}\right) T_{3}+B_{2}\left(\tilde{r}_{2}, \tilde{v}_{3}\right) \geq Q \\
B_{3}\left(\tilde{r}_{1}\right) T_{1}+B_{4}\left(\tilde{r}_{1}, \tilde{v}_{1}\right)+Q_{\mathrm{D}}^{\mathrm{str}}+Q_{\mathrm{D}}^{\mathrm{cir} 2}=Q
\end{array}
$$

In (22), $\tilde{r}_{1}$ and $\tilde{v}_{1}$ are the optimal solutions of (18), and $\tilde{r}_{2}$ and $\tilde{v}_{3}$ are the optimal solutions of (19).

However, the solution of problem (22) is still hard to achieve due to the integer constraint $n$. Then, the following two lemmas are proposed so as to simplify problem (22).

Lemma 2: For problem (22), the optimal solution of the number of the laps in mode 1 can be expressed as

$$
\tilde{n} \in\left\{\left\lfloor\frac{\hat{T}_{1} \hat{v}_{1}}{2 \pi \hat{r}_{1}}\right\rfloor,\left\lceil\frac{\hat{T}_{1} \hat{v}_{1}}{2 \pi \hat{r}_{1}}\right\rceil\right\},
$$

where $\hat{T}_{1}, \hat{v}_{1}$ and $\hat{r}_{1}$ are given in Appendix A.

\section{Proof: See APPENDIX A.}

Lemma 3: Denote by $\tilde{Q}_{R}^{\text {cirl }}$ and $\tilde{Q}_{R}^{\text {str }}$ the optimal data bits received by the UAV in modes 1 and 2, respectively, which can be obtained by solving problem (22) and then substituting the obtained solutions into (15) and (16). Let $\hat{Q}_{\mathrm{R}}^{\text {cirl }}=Q-\tilde{Q}_{\mathrm{R}}^{\text {str }}-B_{1}\left(\tilde{r}_{2}\right) \tilde{T}_{3}-B_{2}\left(\tilde{r}_{2}, \tilde{v}_{3}\right)$. Then, $\tilde{Q}_{\mathrm{R}}^{\text {cirl }}$ can be expressed as

$$
\tilde{Q}_{\mathrm{R}}^{\text {cirl }}=\max \left\{\hat{Q}_{\mathrm{R}}^{\text {cirl }}, Q_{\mathrm{ext}}\right\},
$$

where $Q_{\mathrm{ext}}=2 n \pi \sqrt{\tilde{r}_{1}}\left(\frac{c_{2}+c_{1} g^{2} \tilde{r}_{1}^{2}}{c_{2} g^{2}}\right)^{\frac{1}{4}} \log _{2}\left(1+\frac{P_{\mathrm{S}} \gamma_{0}}{H^{2}+\tilde{r}_{1}^{2}}\right)$.

Proof: By fixing the value of the radius and the speed of mode 1, it can be easily found that the power consumption of the UAV is monotonically decreasing for $Q_{\mathrm{R}}^{\text {cirl }} \in\left(0, Q_{\mathrm{ext}}\right)$ and monotonically increasing for $Q_{\mathrm{R}}^{\text {cirl }} \in\left(Q_{\mathrm{ext}},+\infty\right)$. Since $B_{1}\left(\tilde{r}_{2}\right) \tilde{T}_{3}+B_{2}\left(\tilde{r}_{2}, \tilde{v}_{3}\right)$ is a lower-bound of the data bits received by the UAV relay received in mode $3, \quad \tilde{Q}_{R}^{\text {cirl }} \geq \hat{Q}_{R}^{\text {cirl }}$ must be hold. For the case where $\hat{Q}_{R}^{\text {cirl }} \geq Q_{\text {ext }}$, if $\tilde{Q}_{R}^{\text {cirl }}-\hat{Q}_{R}^{\text {cirl }}>0$ holds, we can always 
find a smaller power consumption by reducing the power consumption of mode 1, while fixing the power consumption of modes 2 and 3 . It contradicts the optimality assumption. So, $\tilde{Q}_{R}^{\text {cirl }}=\hat{Q}_{R}^{\text {cirl }}$ must be hold in this situation. For that case where $\hat{Q}_{\mathrm{R}}^{\text {cirl }}<Q_{\text {ext }}$, if $\left|\tilde{Q}_{\mathrm{R}}^{\text {cirl }}-Q_{\text {ext }}\right|>0$ holds, we can always find a smaller power consumption by reducing the power consumption of mode 1 while fixing the power consumption of modes 2 and 3. It also contradicts the optimality assumption. So, $\tilde{Q}_{\mathrm{R}}^{\text {cirl }}=Q_{\text {ext }}$ must be hold in order to achieve the minimum power consumption. In conclusion, $\tilde{Q}_{\mathrm{R}}^{\text {cirl }}=\max \left\{\hat{Q}_{\mathrm{R}}^{\text {cirl }}, Q_{\text {ext }}\right\}$ holds.

For the case that $\tilde{n}$ is known, problem (22) can be rewritten as the other sub-problem, giving

$$
\begin{gathered}
\left(\tilde{v}_{2}, \tilde{r}_{3}\right)=\underset{v_{2}, T_{3}}{\arg \min } \frac{2 \pi \tilde{r}_{1}}{\tilde{v}_{1}} \tilde{n}\left[\left(c_{1}+\frac{c_{2}}{g^{2} \tilde{r}_{1}^{2}}\right) \tilde{v}_{1}^{3}+\frac{c_{2}}{\tilde{v}_{1}}\right]+\frac{L-\tilde{r}_{1}-\tilde{r}_{2}}{v_{2}}\left(c_{1} v_{2}^{3}+\frac{c_{2}}{v_{2}}\right)+T_{3}\left[\left(c_{1}+\frac{c_{2}}{g^{2} \tilde{r}_{2}^{2}}\right) \tilde{v}_{3}^{2}+\frac{c_{2}}{\tilde{v}_{3}^{2}}\right] \\
\text { s.t. } \quad V_{\min } \leq v_{2} \leq V_{\max }, T_{3} \geq 0 \\
\qquad \frac{2 \tilde{n} \pi \tilde{r}_{1}}{\tilde{v}_{1}} \log _{2}\left(1+\frac{P_{\mathrm{S}} \gamma_{0}}{H^{2}+\tilde{r}_{1}^{2}}\right)+B_{1}\left(\tilde{r}_{2}\right) T_{3}+B_{2}\left(\tilde{r}_{2}, \tilde{v}_{3}\right)+\frac{F_{1}\left(L-\tilde{r}_{2}\right)-F_{1}\left(\tilde{r}_{1}\right)}{v_{2} \ln 2} \geq Q \\
B_{3}\left(\tilde{r}_{1}\right) \tilde{T}_{1}+B_{4}\left(\tilde{r}_{1}, \tilde{v}_{1}\right)+\frac{F_{2}\left(L-\tilde{r}_{1}\right)-F_{2}\left(\tilde{r}_{2}\right)}{v_{2} \ln 2}+T_{3} \log _{2}\left(1+\frac{P_{\mathrm{R}} \gamma_{0}}{H^{2}+\tilde{r}_{2}^{2}}\right)=Q
\end{gathered}
$$

In (25), $\tilde{T}_{1}$ can be achieved by substituting $\tilde{r}_{1}, \tilde{v}_{1}$ and $\tilde{n}$ into (1). It can be observed that the cost function and the constraint set in problem (25) are convex, and thus it is a convex optimization problem. The solution of (25) can be obtained by solving the Karush-Kuhn-Tucker (KKT) equations, and can be expressed as

$$
\begin{gathered}
\tilde{v}_{2}=-0.5 \sqrt{f_{2}}+0.5 \sqrt{-f_{2}+\frac{a_{9} a_{10}}{c_{1} a_{5} \sqrt{f_{2}}\left(L-\tilde{r}_{1}-\tilde{r}_{2}\right)}} \\
\tilde{T}_{3}=\frac{2 f_{2} k a_{10}-4 a_{9} a_{10}^{2} \tilde{v}_{2}-2 \sqrt{f_{2}}\left[a_{10}\left(2 a_{9} a_{10}-k \tilde{v}_{2}\right)-8 c_{2} a_{5}\left(L-\tilde{r}_{1}-\tilde{r}_{2}\right)\left(a_{8}-b_{3} \tilde{T}_{1}\right)\right]}{16 c_{2} a_{5}^{2} \sqrt{f_{2}}\left(L-\tilde{r}_{1}-\tilde{r}_{2}\right)} \\
+\frac{4 c_{1} a_{5} a_{10}\left[f_{2} k-24 c_{2} a_{5}\left(L-\tilde{r}_{1}-\tilde{r}_{2}\right)\right]\left(\sqrt{f_{2}}+\tilde{v}_{2}\right)}{16 c_{2} a_{5}^{2} k}
\end{gathered}
$$

where

$$
k=\left\{3 \sqrt{3} \sqrt{c_{1}^{2} a_{5}^{2}\left(L-\tilde{r}_{1}-\tilde{r}_{2}\right)^{2} c_{1}^{2}\left[27 a_{9}^{4} a_{10}^{4}+4096 c_{1} c_{2}^{3} a_{5}^{4}\left(L-\tilde{r}_{1}-\tilde{r}_{2}\right)^{4}\right]}-27 c_{1} a_{9}^{2} a_{5} a_{10}^{2}\left(L-\tilde{r}_{1}-\tilde{r}_{2}\right)\right\}^{\frac{1}{3}}
$$$$
f_{2}=\frac{8 c_{2} a_{5}\left(L-\tilde{r}_{1}-\tilde{r}_{2}\right)}{k}-\frac{k}{6 c_{1} a_{5}\left(L-\tilde{r}_{1}-\tilde{r}_{2}\right)} \quad, \quad a_{5}=\log _{2}\left(1+\frac{P_{\mathrm{R}} \gamma_{0}}{H^{2}+\tilde{r}_{2}^{2}}\right) \quad, \quad a_{9}=\left(c_{1}+\frac{c_{2}}{g^{2} \tilde{r}_{2}^{2}}\right) \tilde{v}_{3}^{2}+\frac{c_{2}}{\tilde{v}_{3}^{2}}
$$
$a_{10}=\frac{F_{2}\left(L-\tilde{r}_{1}\right)-F_{2}\left(\tilde{r}_{2}\right)}{\ln 2}$.

According to lemma 2 and lemma 3, an iterative algorithm is proposed to solve problem (22), leading to a sub-optimal solution to the propulsion power minimization problem (12). First, $\tilde{r}_{1}, \tilde{v}_{1}, \tilde{r}_{2}$ and $\tilde{v}_{3}$ are acquired by solving (18) and (20) with fixed $Q_{\mathrm{R}}^{\text {cirl }}$ and $Q_{\mathrm{D}}^{\text {cir2 }}$. Second, substituting $\tilde{r}_{1}, \tilde{v}_{1}, \tilde{r}_{2}$ and $\tilde{v}_{3}$ into (22) and then according to lemma 3, $\tilde{Q}_{\mathrm{R}}^{\text {cir1 }}$ can be achieved. Next, set $Q_{\mathrm{R}}^{\text {cir1 }}=\tilde{Q}_{\mathrm{R}}^{\text {cirl }}$ and repeat the first two steps until a convergent solution is found. Then, the algorithm of the sub-optimal solution for the propulsion power 
minimization problem can be given as follows.

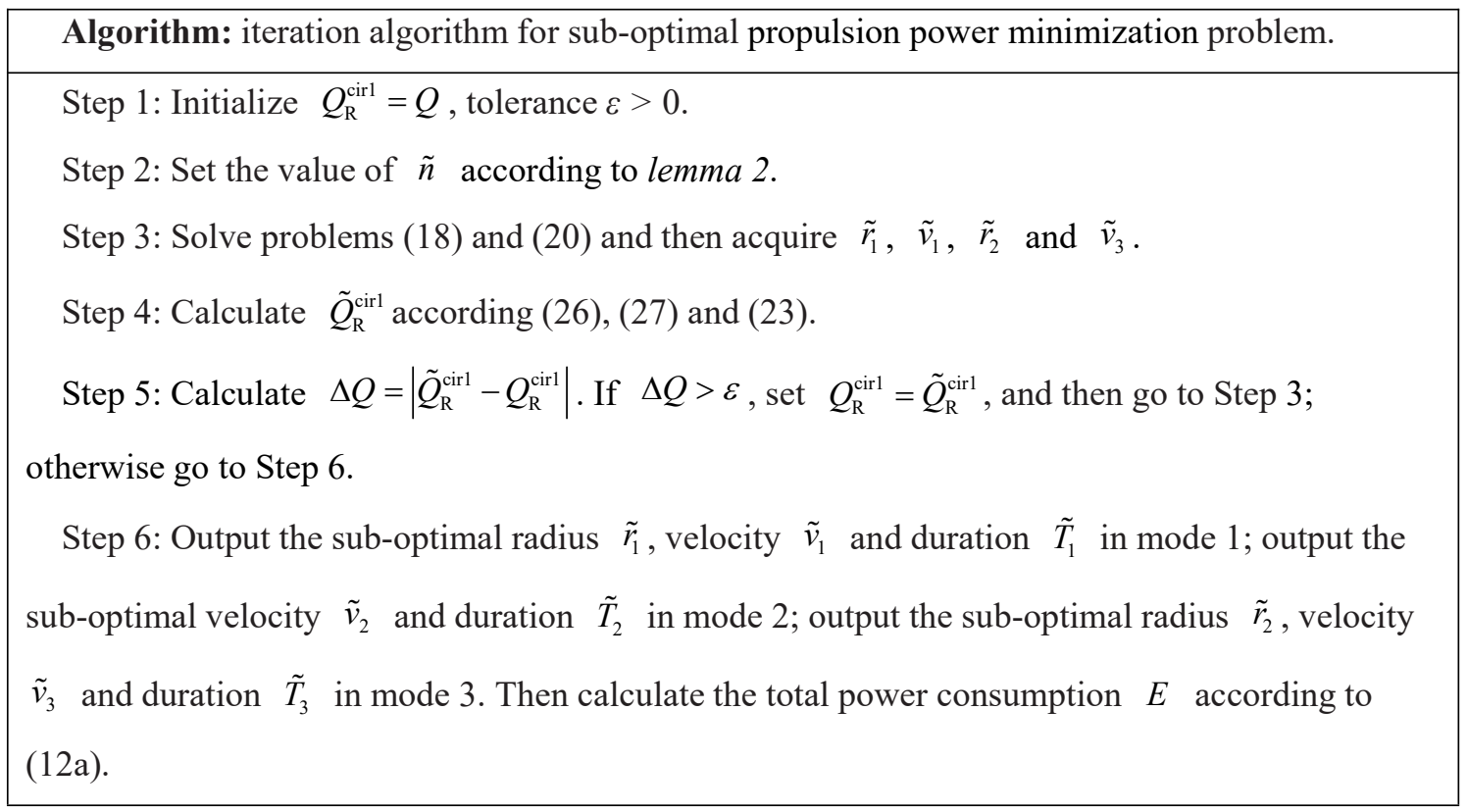

It is worth-mentioning that according to lemma $2, \tilde{n}$ has two possible solutions, i.e., $\left\lfloor\frac{\hat{T}_{1} \tilde{v}_{1}}{2 \pi \tilde{r}_{1}}\right\rfloor$ and $\left\lceil\frac{\hat{T}_{1} \tilde{v}_{1}}{2 \pi \tilde{r}_{1}}\right\rceil$. Therefore, two sets of solution could be achieved if substituting $\tilde{n}=\left\lfloor\frac{\hat{T}_{1} \hat{v}_{1}}{2 \pi \hat{r}_{1}}\right\rfloor$ and $\tilde{n}=\left\lceil\frac{\hat{T}_{1} \hat{v}_{1}}{2 \pi \hat{r}_{1}}\right\rceil$ into the iteration algorithm. Actually, the optimal solution of the algorithm is the one having the smaller power consumption.

\section{Simulation Results and Discussions}

In this section, computer simulations are conducted to validate the proposed HCST design. In order to make comparisons, a straight flight and a circular flight trajectories are included. The UAV altitude is fixed at $H=200 \mathrm{~m}$. The noise power is set to $\delta^{2}=-110 \mathrm{dBm}$ and the reference channel power is set to $\beta_{0}=-50 \mathrm{~dB}$. Furthermore, $c_{1}=9.26 \times 10^{-4}, c_{2}=2250, v_{\min }=10 \mathrm{~m} / \mathrm{s}$ and $v_{\max }=50 \mathrm{~m} / \mathrm{s}$ are assumed.

Fig. 2 makes the comparisons with the sub-optimal trajectory of HCS and optimal trajectory of HCS in terms of the circular radius and the speed of modes 1 and 3. The sub-optimal results are achieved by the iteration algorithm mentioned above. While a search algorithm is used to solve the problem (12) which leads to the optimal results. It is shown in Fig. 1 that the UAV's trajectory and total propulsion power consumption of sub-optimal and optimal are closed.

In Fig. 2, the distance between $\mathrm{S}$ and $\mathrm{D}$ is set to $L=5000 \mathrm{~m}$, the transmit power of S and UAV relay is set to $P_{\mathrm{S}}=P_{\mathrm{R}}=1 \mathrm{~W}$. As shown in Fig. 2 (a) and (b), the circular radius $r_{1}$ is monotonically increasing for $Q \in(200,280)$ while the speed $v_{1}$ is monotonically decreasing for $Q \in(200,280)$. That's because with the increasing of $Q$, the data bits $Q_{\mathrm{R}}^{\text {cirl }}$ transmitted from $\mathrm{S}$ node to UAV node must increase. When a larger $Q_{\mathrm{R}}^{\text {cirl }}$ data bits is need to be transmit from S node to the UAV, the circular radius $r_{1}$ and speed $v_{1}$ should be optimized jointly. Furthermore, a larger radius and lower speed lead to a smaller power consumption. Moreover, the value of circular radius $r_{1}$ and speed $v_{1}$ changed rapidly in $Q \in(280,300)$. The reason is a larger number of lap in mode 
1 can achieve a smaller total power consumption with the increasing of $Q$. Then, the radius $r_{1}$ and speed $v_{1}$ can be derived by updating the number of lap in mode 1 which causing the jump phenomenon. And the number of lap in mode 1 is given in Table 1. Meanwhile, the circular radius $r_{2}$ and speed $v_{3}$ are almost not changed. Since there is no lap constraint in mode 3, and the energy-efficiency trajectory has the potential to achieve the minimum power consumption.

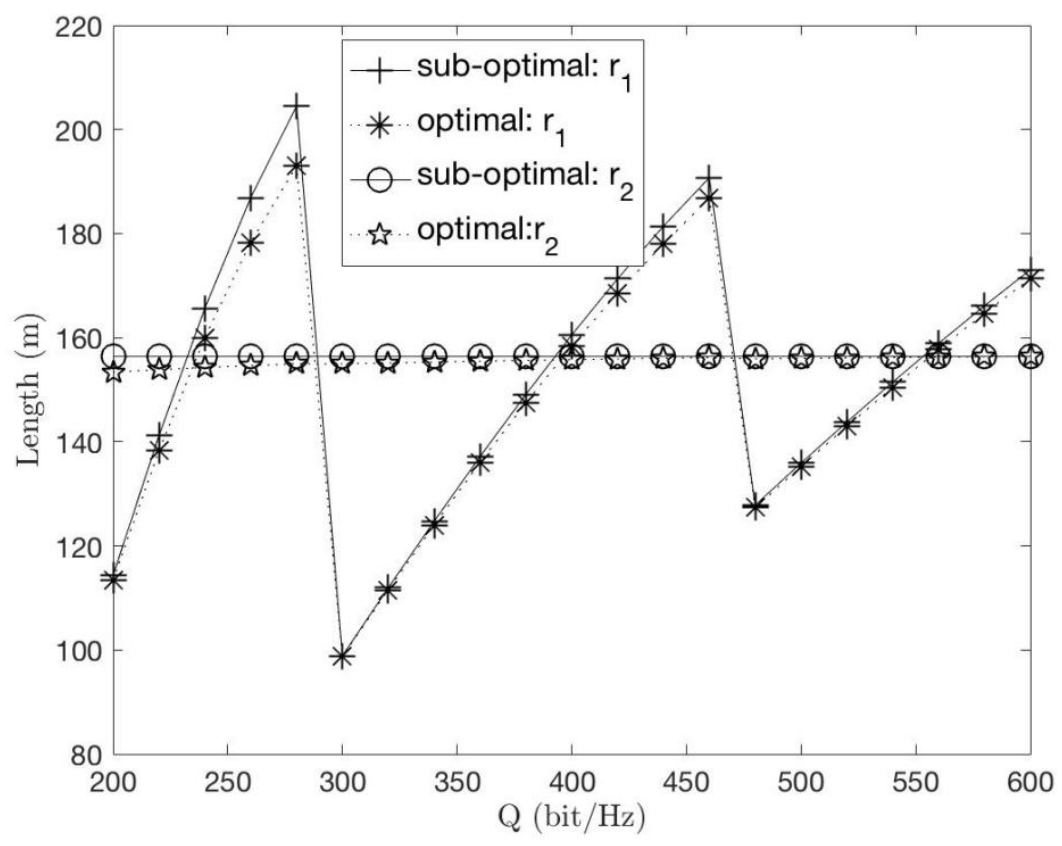

(a)

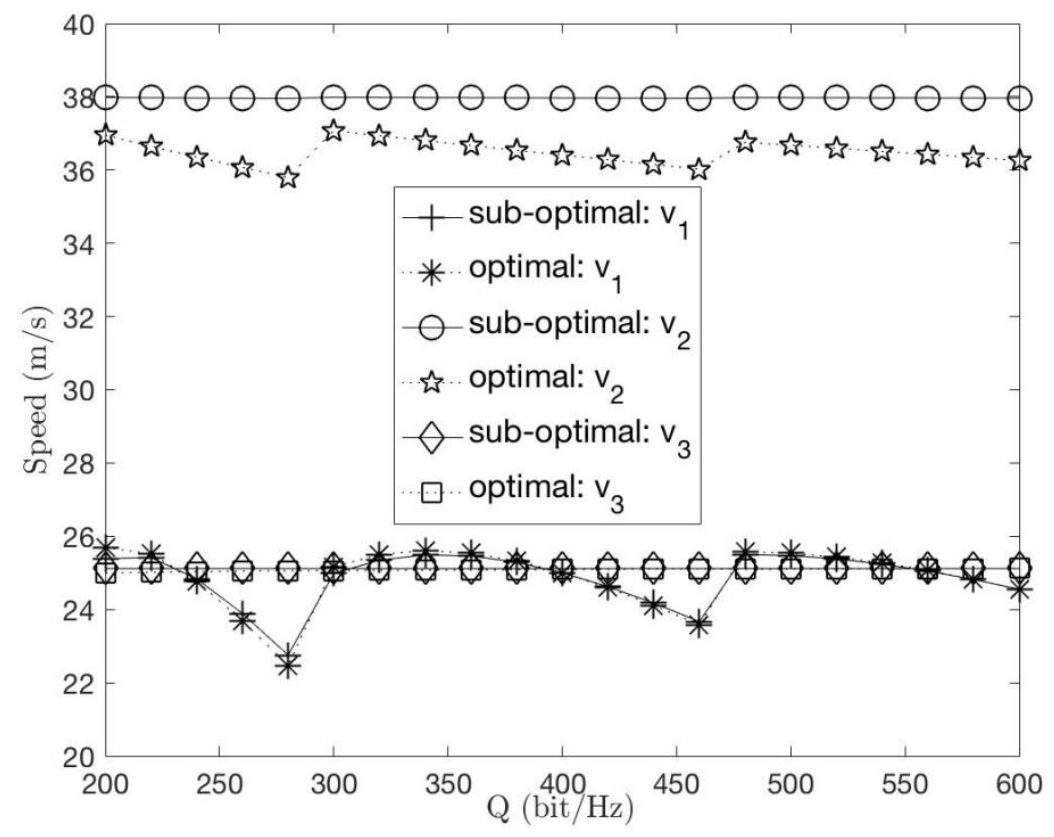

(b)

Fig. 2 Trajectory versus total data bits $Q$

Table 1 The number of laps in mode 1 versus the total data bits $Q$

\begin{tabular}{|c|c|c|c|c|c|c|c|c|c|c|c|}
\hline$Q$ (bit) & 200 & 220 & 240 & 260 & 280 & 300 & 320 & 340 & 360 & 380 & 400 \\
\hline$n$ (laps) & 1 & 1 & 1 & 1 & 1 & 2 & 2 & 2 & 2 & 2 & 2 \\
\hline$Q$ (bit) & 420 & 440 & 460 & 480 & 500 & 520 & 540 & 560 & 580 & 600 & \\
\hline$n$ (laps) & 2 & 2 & 2 & 3 & 3 & 3 & 3 & 3 & 3 & 3 \\
\hline
\end{tabular}




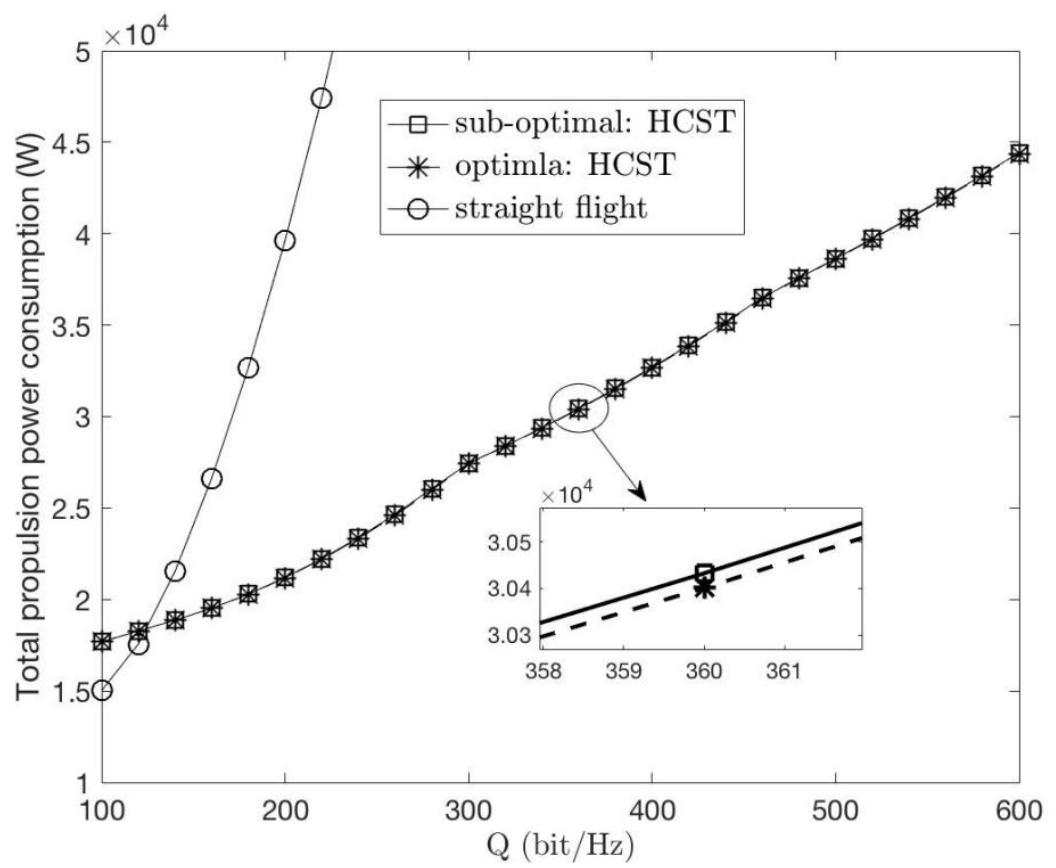

(a)

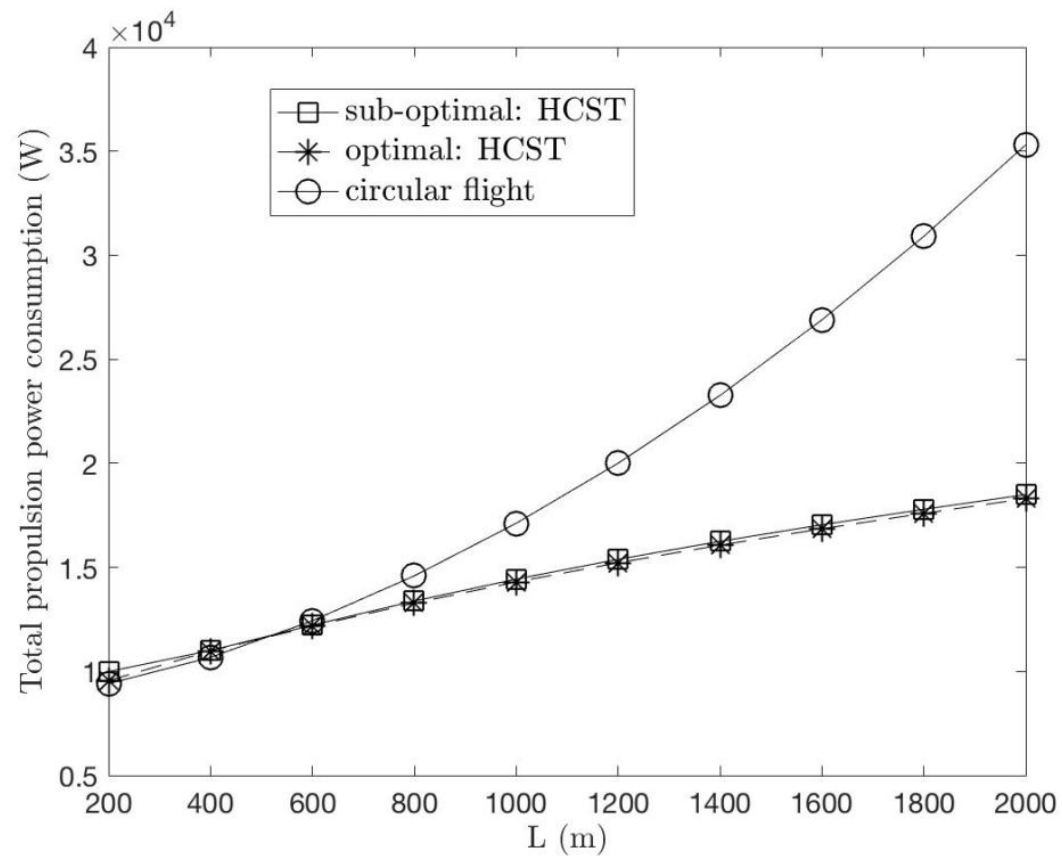

(b)

Fig. 3 Total propulsion power consumption versus total data bits $Q$ and the distance $L$

Figs. 3 make the comparisons with the HCS, straight flight and circular flight trajectory in terms of the total propulsion power consumption. It is shown in Fig. 3 that the HCS perform well in big data and long distance compared to straight and circular trajectory.

In Fig. 3 (a), the distance between $\mathrm{S}$ and $\mathrm{D}$ is set to $L=5000 \mathrm{~m}$, the transmit power of $\mathrm{S}$ and UAV relay is set to $P_{\mathrm{S}}=P_{\mathrm{R}}=1 \mathrm{~W}$. Fig. 3 (a) present the total propulsion power consumption of the HCS and straight flight trajectory, showing that the HCS can saving more energy compare to the straight flight trajectory when the total data bits $Q$ is large. For instance, Fig. 3 (a) shows that the HCS can achieve about $18.4 \mathrm{~kW}$ energy savings compared to the straight flight trajectory when $Q=200$ bit. In Fig. 3 (b), the total data bits is set to $Q=300$ bit, the 
transmit power of $\mathrm{S}$ and UAV relay is set to $P_{\mathrm{S}}=P_{\mathrm{R}}=1 \mathrm{~W}$. Fig. 3 (b) present the total propulsion power consumption of the HCS and circular flight trajectory, showing that the HCS can saving more energy compare to the circular flight trajectory when the distance $L$ is large. For instance, Fig. 3 (b) shows that the HCS can achieve about $2.7 \mathrm{~kW}$ energy savings compared to the circular flight trajectory when $L=1000 \mathrm{~m}$.

\section{Conclusions}

This paper proposes a new HCS trajectory referring to the case of long distance and big data communication. And investigates the unmanned aerial vehicle's (UAV) propulsion power minimization problem subject to the total data bits constraint of a UAV-enabled full-duplex one-way mobile relaying system. Considering a sub-optimal problem, and the corresponding sub-optimal solutions are obtained by an iterative algorithm. The results show that the HCS performs better than straight or circular flight trajectory in terms of energy saving when long distance and big data communication is considered.

\section{Abbreviations}

DF: decode-and-forward; HCST: hybrid circular/straight trajectory; LoS: line-of-sight; KKT: Karush-Kuhn-Tucker; UAV: unmanned aerial vehicle;

\section{Declarations}

\section{Availability of data and materials}

Please contact the corresponding author for data requests.

\section{Competing interests}

The authors declare that they have no competing interests.

\section{Funding}

This work is supported in part by the National Natural Science Foundation of China (Grant No. 61871241), by the Science and Technology Program of Nantong (Grant No. JC2019114), by the Nantong University-Nantong Joint Research Center for Intelligent Information Technology (Grant No. KFKT2017A03).

\section{Authors' contributions}

All the authors take part in the discussion of the work described in this paper. The author Xiaodong Ji wrote the first version of the paper. The author Tao Wang helped Xiaodong Ji revise the paper. The authors Chong Qian and Chenhao Huang did the experiments of the paper. All the four authors worked closely during the preparation and writing of the manuscript. All the authors read and approved the final manuscript.

\section{Acknowledgements}


Not applicable.

\section{References}

[1] Y. Zeng, R. Zhang, and T.J. Lim, "Wireless communications with unmanned aerial vehicles: Opportunities and challenges," IEEE Commun. Mag., vol. 54, no. 5, pp. 36-42, May 2016.

[2] J. Lyu, Y. Zeng, and T.J. Lim, "Placement optimization of UAV-mounted mobile base stations," IEEE Commun. Lett., vol. 21, no. 3, pp. 604-607, Mar. 2017.

[3] C.C. Lai, C.T. Chen, and Y. Wang, "On-demand density-aware UAV base station 3D placement for arbitrarily distributed users with guaranteed data rates," IEEE Wireless Commun. Lett., vol. 8, no. 3, pp. 913-916, Aug. 2019.

[4] M. Mozaffari, W. Saad, and M. Bennis, "Wireless communication using unmanned aerial vehicles (UAVs): Optimal transport theory for hover time optimization," IEEE Trans. on Wireless Commun., vol. 16, no. 12, pp. 8052-8066, Dec. 2017.

[5] S.H. Zhang and H.L. Zhang, "Joint trajectory and power optimization for UAV relay networks," IEEE Commun. Lett., vol. 22, no. 1, pp. 161-164, Jan. 2018.

[6] H. Baek and J. Lim, "Design of future UAV-relay tactical data link for reliable UAV control and situational awareness," IEEE Commun. Mag., vol. 56, no. 10, pp. 144-150, Nov. 2018.

[7] R.F. Fan, J.N. Cui, and S. Jin, "Optimal node placement and resource allocation for UAV relaying network," IEEE Commun. Lett., vol. 22, no. 4, pp. 808-811, Apr. 2018

[8] Y.Q. Zhou, H. Liu, and A.G. Pan, "Cooperative multicast with location aware distributed mobile relay selection: Performance analysis and optimized design," IEEE Trans. on Veh. Technol., vol. 66, no. 9, pp. 8291-8302, Sept. 2017.

[9] J. Lyu, Y. Zeng, and R. Zhang, "Cyclical multiple access in UAV-aided communications: A throughput-delay tradeoff," IEEE Wireless Commun. Lett., vol. 5, no. 6, pp. 600-603, May. 2016.

[10] Y. Zeng and R. Zhang, "Energy-efficient UAV communication with trajectory optimization," IEEE Trans. on Wireless Commun., vol. 16, no. 6, pp. 3747-3760, Jun. 2017.

[11] Y. Zeng, R. Zhang, and T.J. Lim, "Throughput maximization for UAV-enabled mobile relaying systems," IEEE Trans. on Commun., vol. 64, no. 12, pp. 4983-4996, Dec. 2016.

[12] G.C. Zhang, H.Q. Yan, and Y. Zeng, "Trajectory optimization and power allocation for multi-hop UAV relaying communications," IEEE Access, no. 6, pp. 48566-48576, Jun. 2018.

[13] M. Hua, Y. Wang, and Z.M. Zhang, "Outage probability minimization for low-altitude UAV-enabled full-duplex mobile relaying systems," China Commun., vol. 15, no. 5, pp. 9-24, May 2018.

[14] X. Jiang, Z. Wu, and Z.D. Yin, "Power and trajectory optimization for UAV-enabled amplify-and-forward relay networks," IEEE Access, vol. 6, pp. 48688-48696, Jun. 2018.

[15] Z. Xue, J.L. Wang, and G.R. Ding, "Joint 3D location and power optimization for UAV-enabled relaying systems," IEEE Access, vol. 6, pp. 43113-43124, Jun. 2018.

[16] J. Baek, S.I. Han, Y. Han, "Optimal resource allocation for non-orthogonal transmission in UAV relay systems," IEEE Wireless Commun. Lett., vol. 7, no. 3, pp. 356-359, Mar. 2018. 
[17] D.H. Choi, S.H. Kim, and D.K. Sung, "Energy-efficient maneuvering and communication of a single UAV-based relay," IEEE Trans. Aerosp. Electron. Syst., vol. 50, no. 3, pp. 2320-2327, Mar. 2014.

[18] Q.H. Song, and F.C. Zheng, "Energy efficient multi-antenna UAV-enabled mobile relay," China Commun., vol. 15, no. 5, pp. 41-50, May 2018.

[19] D.C. Yang, Q.Q. Wu, and Y. Zeng, "Energy tradeoff in ground-to-UAV communication via trajectory design," IEEE Trans. on Veh. Technol., vol. 67, no. 7, pp. 6721-6726, Jul. 2018.

[20] N. Qi, M. Wang, W. Wang, et al, "Energy efficient full-duplex UAV relaying networks under load carry and delivery scheme," IEEE Access, vol. 64, no. 8, pp. 74349-74358, May 2020.

[21] Y. Zeng, J. Xu and R. Zhang, "Energy minimization for wireless communication with rotary-wing UAV," IEEE Trans. Wireless Commun., vol. 18, no. 4, pp. 2329-2345, Apr. 2019.

[22] J.W. Zhang, Y. Zeng, and R. Zhang, "Spectrum and energy efficiency maximization in UAV-enabled mobile relaying," in Proc. IEEE ICC 2017, Paris, France, May 2017, pp. 1-6.

\section{Appendix A}

For problem (22), it is readily to find the total propulsion power consumption is monotonically decreasing at first, and then monotonically increasing for $n$. Firstly, we ignore the constraint that the UAV has to fly the whole laps in mode 1 . Then, the sub-optimal trajectory of mode 1 can be derived by solving problem (28).

$$
\begin{aligned}
& \left(\hat{r}_{1}, \hat{v}_{1}\right)=\arg \min _{r_{1}, v_{1}} \frac{\left(c_{1}+\frac{c_{2}}{g^{2} r_{1}^{2}}\right) v_{1}^{3}+\frac{c_{2}}{v_{1}}}{\log _{2}\left(1+\frac{P_{\mathrm{S}} \gamma_{0}}{H^{2}+r_{1}^{2}}\right)} \\
& \text { s.t. } \quad v_{\text {min }} \leq v_{1} \leq v_{\max }, 0<r_{1}<L
\end{aligned}
$$

The minimum power on the numerator of (28a) can be achieved when (29) holds.

$$
\hat{v}_{1}=\left\{\begin{array}{l}
v_{\min }, \text { if } v_{\min }>\left(\frac{c_{2}}{3 c_{1}+\frac{3 c_{2}}{g^{2} r_{2}^{2}}}\right)^{\frac{1}{4}} \\
\left(\frac{c_{2}}{3 c_{1}+\frac{3 c_{2}}{g^{2} r_{1}^{2}}}\right)^{\frac{1}{4}}, \text { if } v_{\min } \leq\left(\frac{c_{2}}{3 c_{1}+\frac{3 c_{2}}{g^{2} r_{1}^{2}}}\right)^{\frac{1}{4}} \leq v_{\max } . \\
v_{\max }, \text { if } v_{\max }<\left(\frac{c_{2}}{3 c_{1}+\frac{3 c_{2}}{g^{2} r_{1}^{2}}}\right)^{\frac{1}{4}}
\end{array}\right.
$$

Substituting (29) into (28) and then the optimal solution of (28) can be obtained by using one-dimensional search over $0<r_{1}<L$. Then, problem (22) can be written as a convex optimization problem as follows. 


$$
\begin{array}{cl}
\left(\hat{v}_{2}, \hat{T}_{1}, \hat{T}_{3}\right)=\underset{v_{2}, T_{1}, T_{3}}{\arg } T_{1}\left[\left(c_{1}+\frac{c_{2}}{g^{2} \hat{r}_{1}^{2}}\right) \hat{v}_{1}^{2}+\frac{c_{2}}{\hat{v}_{1}^{2}}\right]+\frac{L-\hat{r}_{1}-\tilde{r}_{2}}{v_{2}}\left(c_{1} v_{2}^{3}+\frac{c_{2}}{v_{2}}\right)+T_{3}\left[\left(c_{1}+\frac{c_{2}}{g^{2} \tilde{r}_{2}^{2}}\right) \tilde{v}_{3}^{2}+\frac{c_{2}}{\tilde{v}_{3}^{2}}\right] \\
\text { s.t. } \quad V_{\min } \leq v_{2} \leq V_{\max } \\
\quad T_{1} \geq 0, T_{3} \geq 0 \\
& Q_{\mathrm{R}}^{\text {cirl }}+Q_{\mathrm{R}}^{\mathrm{str}}+B_{1}\left(\tilde{r}_{2}\right) T_{3}+B_{2}\left(\tilde{r}_{2}, \tilde{v}_{3}\right) \geq Q \\
& B_{3}\left(\tilde{r}_{1}\right) T_{1}+B_{4}\left(\tilde{r}_{1}, \tilde{v}_{1}\right)+Q_{\mathrm{D}}^{\mathrm{st}}+Q_{\mathrm{D}}^{\mathrm{cir} 2}=Q
\end{array}
$$

The solution of problem (30) can be obtained by solving the KKT equations, and the optimal value of $\tilde{n}$ can be given as

$$
\tilde{n}=\frac{\hat{T}_{1} \hat{v}_{1}}{2 \pi \hat{r}_{1}}
$$

$$
\hat{T}_{1}=\left\{-192 \times 2^{1 / 3} a_{2}^{2}\left(a_{4} a_{5}-a_{6} b_{1}\right)\left(a_{3} a_{5}-b_{1} b_{3}\right)^{2} c_{1} c_{2} \sqrt{f_{1}}\left(\sqrt{f_{1}}+v_{2}\right)+2\left(a_{4} a_{5}-a_{6} b_{1}\right) k_{1}\left(\sqrt{f_{1}}+v_{2}\right)\right.
$$

Here, $\times\left[-4\left(a_{9} a_{3} a_{6}+a_{1} a_{4} a_{5}-a_{1} a_{6} b_{1}-a_{9} a_{4} b_{3}\right)+2^{2 / 3} \sqrt{f_{1}} k_{1}\right]+8 a_{2}\left(a_{3} a_{5}-b_{1} b_{3}\right) \sqrt{f_{1}} k_{1}\left[4 a_{5} a_{7} c_{2}+a_{4} a_{5} c_{1} f_{1}\left(\sqrt{f_{1}}+v_{2}\right)\right.$

$$
\left.\left.-4 b_{1} a_{8} c_{2}-a_{6} b_{1} c_{1} f_{1}\left(\sqrt{f_{1}}+v_{2}\right)\right]\right\} /\left[32 a_{2}\left(a_{3} a_{5}-b_{1} b_{3}\right)^{2} c_{2} \sqrt{f_{1}} k_{1}\right]
$$

,where

$$
\begin{gathered}
\hat{v}_{2}=-1 / 2 \times \sqrt{f_{1}}+1 / 2 \times \sqrt{\left(a_{1} a_{4} a_{5}+a_{9} a_{3} a_{6}-a_{1} a_{6} b_{1}-a_{9} a_{4} b_{3}\right) /\left[a_{2}\left(a_{3} a_{5}-b_{1} b_{3}\right) c_{1} \sqrt{f_{1}}\right]-f_{1}}, \\
a_{1}=\left(c_{1}+\frac{c_{2}}{g^{2} \hat{r}_{1}^{2}}\right) \hat{v}_{1}^{2}+\frac{c_{2}}{\hat{v}_{1}^{2}}, \\
a_{2}=L-\hat{r}_{1}-\tilde{r}_{2}, \\
a_{3}=\log _{2}\left(1+\frac{P_{\mathrm{S}} \gamma_{0}}{H^{2}+\hat{r}_{1}^{2}}\right), \\
a_{4}=\frac{F_{1}\left(L-\tilde{r}_{2}\right)-F_{1}\left(\hat{r_{1}}\right)}{\ln 2}, \\
a_{5}=\log _{2}\left(1+\frac{P_{\mathrm{R}} \gamma_{0}}{H^{2}+\tilde{r}_{2}^{2}}\right), \\
a_{6}=\frac{F_{2}\left(L-\hat{r}_{1}\right)-F_{2}\left(\tilde{r}_{2}\right)}{\ln 2}, \\
a_{7}=Q-b_{2}, \\
a_{8}=Q-b_{4}, \\
a_{9}=\left(\begin{array}{c}
c_{2} \\
\left.c_{1}+\frac{c_{2}}{g^{2} \tilde{r}_{2}^{2}}\right) \tilde{v}_{3}^{2}+\frac{c_{2}}{\tilde{v}_{3}^{2}}
\end{array}\right.
\end{gathered}
$$

Recall that the UAV has to fly the whole laps in mode 1, namely, $\tilde{n}$ should be an integer, the optimal laps in modea shall be $\left\lfloor\frac{\hat{T}_{1} \hat{v}_{1}}{2 \pi \hat{r}_{1}}\right\rfloor$ or $\left\lceil\frac{\hat{T}_{1} \hat{v}_{1}}{2 \pi \hat{r}_{1}}\right\rceil$. 


\section{Figure legends}

Fig. 1 UAV-enabled mobile relaying system with hybrid circular/straight trajectory

Fig. 2 Trajectory versus total data bits $Q$

Fig. 3 Total propulsion power consumption versus total data bits $Q$ and the distance $L$ 